\title{
Cerebral Blood Flow and Its Connectivity Deficits in Mild Traumatic Brain Injury at the Acute Stage
}

\author{
Fengfang Li, Liyan Lu, Song'an Shang, Huiyou Chen, Peng Wang, Nasir Ahmad Haidari, \\ Yu-Chen Chen $\mathbb{D}$, and Xindao Yin
}

Department of Radiology, Nanjing First Hospital, Nanjing Medical University, Nanjing, China

Correspondence should be addressed to Yu-Chen Chen; chenyuchen1989@126.com and Xindao Yin; y.163yy@163.com

Received 19 March 2020; Revised 30 May 2020; Accepted 8 June 2020; Published 1 July 2020

Academic Editor: Sergio Bagnato

Copyright (c) 2020 Fengfang Li et al. This is an open access article distributed under the Creative Commons Attribution License, which permits unrestricted use, distribution, and reproduction in any medium, provided the original work is properly cited.

\begin{abstract}
Objective. The influence of cognitive impairment after mild traumatic brain injury (mTBI) on cerebral vascular perfusion has been widely concerned, yet the resting-state cerebral blood flow (CBF) connectivity alterations based on arterial spin labeling (ASL) in mild traumatic brain injury (mTBI) remain unclear. This study investigated region CBF and CBF connectivity features in acute mTBI patients, as well as the associations between CBF changes and cognitive impairment. Materials and Methods. Forty-five acute mTBI patients and 42 health controls underwent pseudocontinuous arterial spin labeling (pCASL) perfusion magnetic resonance imaging (MRI). The alterations in regional $\mathrm{CBF}$ and relationship between the $\mathrm{CBF}$ changes and cognitive impairment were detected. The ASL-CBF connectivity of the brain regions with regional CBF significant differences was also compared between two groups. Neuropsychological tests covered seven cognitive domains. Associations between the CBF changes and cognitive impairment were further investigated. Results. Compared with the healthy controls, the acute mTBI patients exhibited increased CBF in the bilateral inferior temporal gyrus (ITG) and decreased CBF in the right middle frontal gyrus (MFG), the bilateral superior frontal gyrus (SFG), and the right cerebellum posterior lobe (CPL). In the mTBI patients, significant correlations were identified between the CBF changes and cognitive impairment. Importantly, the acute mTBI patients exhibited $\mathrm{CBF}$ disconnections between the right CPL and right fusiform gyrus (FG) as well as bilateral ITG, between the left SFG and left middle occipital gyrus (MOG), and between the right SFG and right FG as well as right parahippocampal gyrus. Conclusion. Our results suggest that acute mTBI patients exhibit both regional CBF abnormalities and CBF connectivity deficits, which may underlie the cognitive impairment of the acute mTBI patients.
\end{abstract}

\section{Introduction}

Mild traumatic brain injury (mTBI), also commonly referred to as concussion, is recognized as a major public health concern worldwide [1]. A large number of mTBI patients may have measurable deficits in some cognitive areas, mainly including visuospatial and executive functioning, attention, language, and memory $[2,3]$, which can interfere with daily life. It is estimated that up to $40-50 \%$ of mTBI patients still have cognitive deficit symptoms 3 months after injury and $10-15 \%$ at even years after injury [4]. However, the specific mechanism that leads to brain deficits and ultimately cognitive impairment in mTBI patients is still controversial and remains to be elucidated. In addition to structural and functional brain changes, imaging studies using single-photon emission computed tomography (SPECT) and positron emission tomography (PET) have shown abnormal cerebral blood flow (CBF) and cerebral glucose metabolism in multiple brain regions of mTBI patients at different stages $[5,6]$. Furthermore, there are also indications that alterations of the $\mathrm{CBF}$ are associated with persistent symptoms and impaired cognitive functioning [7]. Meanwhile, several resting-state $\mathrm{CBF}$ alterations have been associated with the core clinical cognitive impairments symptoms of mTBI $[8$, 9]. Although SPECT, PET, and arterial spin labeling (ASL) can all evaluate brain hemodynamics, SPECT and PET are nuclear medicine techniques that require using invasive radioactive tracers, which limit repeated testing. On the contrary, ASL provides a noninvasive means for estimating $\mathrm{CBF}$, which has been shown to be a robust method of 
assessing microvascular perfusion, and is less influenced by pathologic damage to the blood-brain barrier, including TBI [10].

Profiting from a short acquisition time and endogenous contrast agent, ASL perfusion sequence is used to evaluate $\mathrm{CBF}$ at resting-state and could serve as a marker of functional activation albeit, which achieves a direct measure of regional $\mathrm{CBF}$ and independent of complicated calculations [11, 12]. Using this technique, the alterations of CBF in TBI have been explored in animal models [12] and human studies [13], but few studies focused on mTBI, especially the acute mTBI. Recently, chronic TBI [13] and chronic mTBI [14, 15] studies confirmed that the relative disturbances of regional CBF were successfully detected by ASL. Furthermore, Ge et al. attempted to evaluate regional $\mathrm{CBF}$ changes after chronic mTBI by a resting-state ASL and found significant reductions in mean bilateral thalamic $\mathrm{CBF}$, which was significantly correlated with the simultaneous neuropsychological tests, including measures of learning and memory, response and processing speed, and executive function and verbal fluency [16]. However, the CBF changes in multiple brain regions and the association with neuropsychological cognitive results differ largely across studies. In addition to the limited sample size and the large differences among the subjects, an important technical reason may be the influence of partial volume effect (PVE) [17]. Although PVE was considered in a few PET, SPECT, or ASL studies in mTBI, most studies did not include correction for gray matter volume (GMV), leading to biased results of decreased glucose metabolism and $\mathrm{CBF}$ in mTBI patients.

As a reflection of neuronal activity, the CBF in different regions of the brain is not independent. Instead, the $\mathrm{CBF}$ in brain regions from the same functional network may change synchronously to achieve the network's function [18]. Melie-Garcia et al. showed that the highest synchronous fluctuations of CBF occur between homologous cortical regions, and functional networks constructed by $\mathrm{CBF}$ connectivity have similar network characteristics as functional networks constructed by anatomical or functional connectivity [19]. The alterations of ASL-CBF connectivity have been explored in healthy subjects [20] and diverse diseases, such as schizophrenia [21] and autism spectrum disorders [22]. However, to our knowledge, no studies have evaluated the application of ASL-CBF connectivity in mTBI, and the ASL-CBF connectivity alterations in mTBI patients remain unknown.

In the current study, we utilized ASL-MRI to clarify the pattern of $\mathrm{CBF}$ changes and the associations between $\mathrm{CBF}$ changes and cognitive impairment in acute mTBI patients. Furthermore, we investigated whether the brain region with abnormal $\mathrm{CBF}$ also exhibited $\mathrm{CBF}$ connectivity changes in acute mTBI patients. Specifically, to exclude the effect of cortical atrophy on the CBF results, we used information from coregistered structural MRI data to correct ASL-MRI data as well as for GMV variations. We hypothesized that the acute $\mathrm{mTBI}$ patients would have different cerebral perfusion and $\mathrm{CBF}$ connectivity compared with healthy control (HC), and these changes may be associated with cognitive impairment after mTBI.

\section{Materials and Methods}

2.1. Participants and Procedure. The investigation was approved by the Ethics Committee of Nanjing Medical University. All individuals provided informed written consent for their clinical data to be used for the research purposes in the study.

A total of 50 patients with mTBI (recruited from the Emergency Department of Nanjing First Hospital) and 44 HC subjects were included in our study. The inclusion criteria were as follows: (1) age (18-60 years) and righthandedness; (2) a history of a closed head injury with posttraumatic amnesia of less than 24-hour duration, no more than 30 minutes of loss of consciousness, or recorded changes of mental status (i.e., dazed, confused, and disoriented); and (3) an initial Glasgow Coma Score (GCS) of 13-15 obtained by the emergency or ambulatory care staff after injury. The exclusion criteria were as follows: (1) a poor quality of the imaging data, (2) MRI contraindications, (3) neuropsychological disease before injury, (4) history of drug or alcohol abuse, and (5) prior brain injury or other neurologic disease (i.e., epilepsy, stroke, and somatic disorders) that would affect the study results. Specially, all mTBI patients were required to undergo CT scans as a part of their clinical evaluation. Based on these criteria, five patients and two healthy subjects were excluded. Thus, 45 patients with clinically defined mTBI (23 males and 22 females, age range: 20-60 years) were included in this study, with a mean interval of 2.79 days (range: 0-7 days) between trauma and MR imaging findings. All of $42 \mathrm{HC}$ subjects ( 19 males and 23 females, age range: 20-59 years) were finally enrolled, and they were confirmed to have no brain diseases according to patient history and imaging. The two groups had no significant demographic differences in age, sex, or education level. The clinical neurocognitive state of all participants of psychosis was quantified with the Montreal Cognitive Assessment (MoCA) [23] that evaluated several aspects of cognitive function, including visuospatial/execution, attention, naming, language, abstraction, memory (short-term immediate and deferred recall), and localization [24]. All subjects underwent the same MRI scan and cognitive function assessment within seven days postinjury. The demographic characteristics and outcome clinical measurements of mTBI and HC subject groups are shown in Table 1.

2.2. MRI Data Acquisition. MRI was performed using a 3.0-T MR system (Ingenia, Philips Medical Systems, Netherlands) and an eight-channel digital head coil receiver. During the MRI scans, earplugs were used to reduce scanner noise, the tight but comfortable foam padding was used to minimize head motion, and participants were instructed to close their eyes and rest peacefully. The resting-state perfusion imaging was performed using a 2D-pseudocontinuous arterial spin labeling (pCASL) sequence (repetition time $=4000 \mathrm{~ms}$; echo time $=11 \mathrm{~ms}$; label duration $=1650 \mathrm{~ms}$; postlabel delay = $1600 \mathrm{~ms}$; flip angle $=90^{\circ}$; field of view $=240 \mathrm{~mm} \times 240 \mathrm{~mm}$; slice thickness $=5 \mathrm{~mm}$ with $10 \%$ gap; matrix $=64 \times 64 ; 20$ axial slices; total scan duration $=4 \mathrm{~min}$ and $08 \mathrm{~s}$ ). Finally, each subject contained 60 volumes used as 30 label-control 
TABLE 1: Demographic and clinical characteristics of the mTBI patients and healthy controls.

\begin{tabular}{lccc}
\hline Characteristic & mTBI $(n=45)$ & Controls $(n=42)$ & $p$ value \\
\hline Age (years) & $41.36 \pm 10.74$ & $40.57 \pm 9.95$ & 0.725 \\
Education (years) & $12.82 \pm 2.75$ & $13.00 \pm 2.96$ & 0.773 \\
Sex (female/male) & $22 / 23$ & $23 / 19$ & 0.669 \\
MoCA scores & $23.67 \pm 2.77$ & $26.21 \pm 1.79$ & $<0.001^{*}$ \\
Visuospatial/executive & $3.49 \pm 1.27$ & $4.19 \pm 1.06$ & $0.007^{*}$ \\
Naming & $2.82 \pm 0.38$ & $2.86 \pm 0.35$ & 0.662 \\
Attention & $5.40 \pm 0.86$ & $5.86 \pm 0.42$ & $0.003^{*}$ \\
Language & $2.22 \pm 0.73$ & $2.62 \pm 0.54$ & $0.005^{*}$ \\
Abstraction & $1.56 \pm 0.58$ & $1.71 \pm 0.45$ & 0.165 \\
Memory & $2.47 \pm 1.29$ & $2.98 \pm 1.33$ & 0.074 \\
Orientation & $5.71 \pm 0.45$ & $5.83 \pm 0.38$ & 0.180 \\
\hline
\end{tabular}

The data are shown as the mean \pm SD. mTBI: mild traumatic brain injury; MoCA: Montreal Cognitive Assessment. ${ }^{*} p<0.05$.

image pairs. Sagittal 3D T1-weighted images were acquired using a three-dimensional turbo fast echo (3D-TFE) T1WI sequence (repetition time $=8.1 \mathrm{~ms}$; echo time $=3.7 \mathrm{~ms}$; flip angle $=8^{\circ}$; field of view $=256 \mathrm{~mm} \times 256 \mathrm{~mm}$; acquisition matrix $=256 \times 256$; slice thickness $=1 \mathrm{~mm}$; gap $=0 \mathrm{~mm} ; 172$ sagittal slices; total scan duration $=5 \mathrm{~min}$ and $28 \mathrm{~s}$ ).

2.3. MRI Data Processing and CBF Calculation. The PCASL data was processed to generate CBF maps using ASL data processing toolbox: ASLtbx (https://cfn.upenn.edu/ zewan) [25]. The detailed CBF maps calculation procedures have been described in the previous study [26, 27]. Statistical parameter mapping software (SPM8) (http://www.fil.ion.ucl .ac.uk/spm/software/spm8/) and ASL data processing toolbox were used to analyze the ASL image data. Control and label ASL images were rearranged and adjusted to correct head movement. The SPM8 software was used to perform a nonlinear transformation on the CBF images of $42 \mathrm{HC}$ subjects, which were coregistered with the PET-perfusion template in the Montreal Neurological Institute (MNI) space. The MNI-standard CBF template was defined as the average coregistered CBF images of $42 \mathrm{HC}$ subjects. The CBF images of all participants, including mTBI patients and HC subjects, were then coregistered to the MNI-standard CBF template. Each coregistered CBF was removed from the nonbrain tissue and spatially smoothed with a GAUSSIAN of $8 \mathrm{~mm} \times 8 \mathrm{~mm} \times 8 \mathrm{~mm}$ FWHM. Normalization was performed by dividing the cerebral blood flow per voxel by the average cerebral blood flow across the entire brain [28].

2.4. GMV Calculation. The GMV of each voxel was calculated using SPM8. The structural MR images were segmented into white matter (WM), gray matter (GM), and cerebrospinal fluid (CSF) using the standard uniform segmentation model. The segmented images were used to create a custom DARTEL template, which was then normalized to the Montreal Neurological Institute (MNI) space. Then, the exponential lie algebra (DARTEL) technique was used to perform nonlinear deformation of GM concentration images and resampling them to the voxel size of $1.5 \mathrm{~mm} \times 1.5 \mathrm{~mm} \times$
$1.5 \mathrm{~mm}$ [29]. The GMV of each voxel was obtained by multiplying the GM concentration graph by the nonlinear determinant obtained by the spatial normalization step. Then, the GMV images were smoothed with a Gaussian kernel of $6 \mathrm{~mm} \times 6 \mathrm{~mm} \times 6 \mathrm{~mm}$ FWHM. Finally, after spatial preprocessing of the data, the normalized, modulated, and smooth GMV maps were used for statistical analysis.

2.5. CBF Connectivity Analyses. Referring to the previous research methods on CBF connectivity [19], in order to detect whether CBF connectivity abnormalities also exist in brain regions with CBF changes in mTBI patients, the clusters with significant group differences in the $\mathrm{CBF}$ were selected as seed (regions of interest) ROIs. The CBF values for each ROI within each subject were extracted from a separate CBF map. For each group, a multiple regression model was used to calculate the CBF connections between each ROI seed and all other voxels in the entire brain between individuals with gender and age as confounding covariates. These statistical analyses were used to identify voxels in each group with a positive or negative correlation between $\mathrm{CBF}$ values and $\mathrm{CBF}$ values for each seed ROI. Multiple comparisons were corrected using FDR correction $(p<0.01)$. For each $\mathrm{ROI}$, the CBF-connected graphs of the two groups were merged into a spatial mask, and the CBF of each voxel was then related to the $\mathrm{CBF}$ of the two sets of ROI. For any pair of voxels, the CBF correlation between the two groups may have different slopes, reflecting the difference in CBF connectivity between the two groups. To map voxels that show significantly different CBF correlations between the acute mTBI patients and $\mathrm{HC}$ subjects for each seed ROI, a specific T comparison was established within the spatial mask of the CBF connectivity map of ROI. Here, the specific $t$-comparison refers to a two-sample $t$-test analysis of the differences in CBF connectivity between each ROI and all other voxels in the brain in the combined spatial mask between mTBI and the control group.

2.6. Correlations Analysis. To investigate the relationships between clinical cognitive parameters of mTBI patients and 


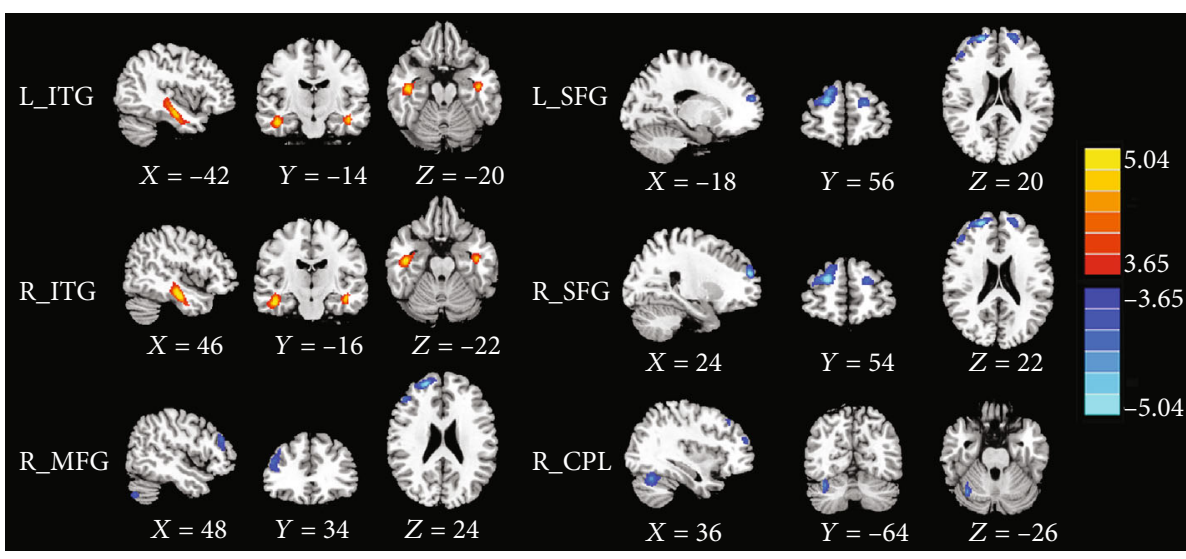

FIgure 1: The CBF differences between the mTBI patients and the HC subjects. Compared with HC, the acute mTBI patients showed increased $\mathrm{CBF}$ in the bilateral inferior temporal gyrus (ITG) as well as decreased CBF in the bilateral superior frontal gyrus (SFG), right middle frontal gyrus (MFG), and right cerebellum posterior lobe (CPL).

regional CBF values and CBF connectivity, the ROI-based correlation analysis with cognitive assessment scores was performed for the mTBI patient group using Pearson's correlation analysis, corrected for age, sex, and educational level. The significance threshold of correlation analysis was set at $p<0.05$.

2.7. Statistical Analysis. Two-sample $t$-tests and $\chi^{2}$ tests were used to analyze the differences in the demographic and clinical data between the mTBI patients and healthy controls by the SPSS 19.0 software package $(p<0.05$ was considered to be significant). For the normalized CBF and CBF connectivity analyses, a group comparison between the $\mathrm{HC}$ group and mTBI group was performed using a twosample $t$-test, corrected for age, sex, and education level. The significance statistical threshold was set as $p<0.01$, using false discovery rate (FDR) correct. For each subject, normalized CBF of each cluster with significant differences between groups was extracted for region of interest- (ROI-) based analysis. In order to exclude the influence of GMV on the CBF comparison, we repeated the voxel-based CBF analysis by taking the GMV of each voxel as an uninterested covariable.

\section{Results}

3.1. Participants and Clinical Data. Demographics and clinical cognitive assessment of the subjects are summarized in Table 1. Differences in age, sex, and education level between groups were not significant (all $p>0.05$ ). In this study, the major mechanism of trauma was motor vehicle collision injury (traffic accidents) [20 of 45 patients (44.4\%)], followed by fall from variable heights which was the last [17 of 45 patients $(37.8 \%)$ ] and assault [ 8 of 45 patients (17.8\%)]. As expected, acute mTBI patients had significantly lower MoCA scores than HC subjects $(p<0.001)$. Among all subcategories of the MoCA, only the visuospatial/executive $(p=0.007)$, attention $(p=0.003)$, and language $(p=0.005)$ scores in the mTBI group were significantly lower than those in the HC group, but score differences of abstraction, memory, naming,
TABLE 2: Brain regions with significant group differences in normalized CBF.

\begin{tabular}{lcccc}
\hline $\begin{array}{l}\text { Brain } \\
\text { regions }\end{array}$ & BA & $\begin{array}{c}\text { Peak MNI coordinates } \\
x, y, z(\mathrm{~mm})\end{array}$ & $\begin{array}{c}\text { Peak } T \\
\text { value }\end{array}$ & $\begin{array}{c}\text { Cluster size } \\
\text { (voxels) }\end{array}$ \\
\hline $\begin{array}{c}\text { mTBI > HC } \\
\text { L_ITG }\end{array}$ & 20 & $-42,-14,-20$ & 4.986 & 472 \\
R_ITG & 20 & $46,-16,-22$ & 5.319 & 616 \\
mTBI < HC & & & & \\
R_MFG & 46 & $48,34,24$ & -4.361 & 332 \\
L_SFG & 10 & $-18,56,20$ & -4.499 & 142 \\
R_SFG & 10 & $24,54,22$ & -5.299 & 404 \\
R_CPL & - & $36,-64,-26$ & -4.514 & 276 \\
\hline
\end{tabular}

Thresholds were set at a corrected $p<0.01$ corrected by FDR criterion. MNI: Montreal Neurological Institute; mTBI: mild traumatic brain injury; HC: healthy control; ITG: inferior temporal gyrus; SFG: superior frontal gyrus; MFG: middle frontal gyrus; CPL: cerebellum posterior lobe.

and orientation between mTBI patients and HC group were not significant $(p>0.05)$.

3.2. Group Differences in Resting-State Normalized CBF. The CBF differences between the mTBI patients and the HC subjects are shown in Figure 1 and Table 2. In voxel-based analysis, the acute mTBI patients showed increased CBF in the bilateral inferior temporal gyrus (ITG) compared with HC. In contrast, these patients also had significantly decreased $\mathrm{CBF}$ in the bilateral superior frontal gyrus (SFG), right middle frontal gyrus (MFG), and right cerebellum posterior lobe (CPL).

The distribution of the brain regions with significant differences in the CBF after GMV correction is shown in Figure 2 and Table 3. After GMV correction, the acute mTBI patients exhibited significantly increased $\mathrm{CBF}$ in the bilateral ITG and decreased CBF in the bilateral SFG (including part of right MFG region), which were the same as the regions without GMV correction. However, the original cluster with reduced CBF in the right CPL disappeared and a new cluster 


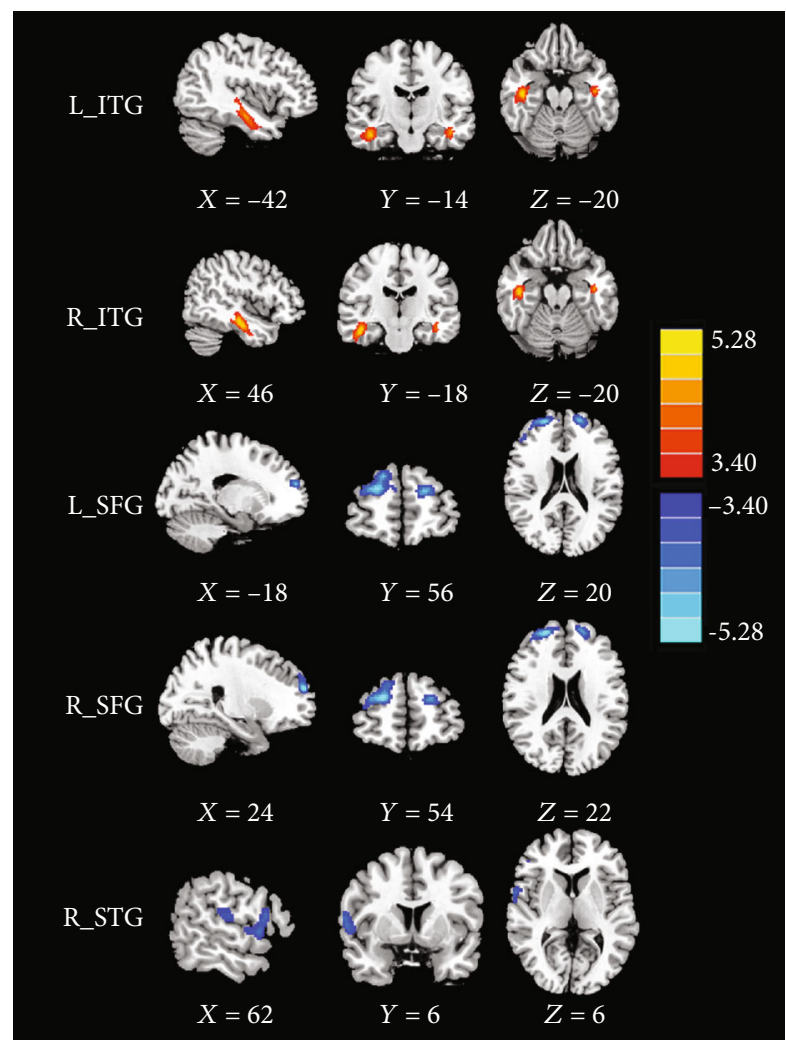

Figure 2: The CBF differences between the mTBI patients and the HC subjects after GMV correction. Compared with HC, the acute mTBI patients showed increased $\mathrm{CBF}$ in the bilateral inferior temporal gyrus (ITG) as well as decreased CBF in the bilateral superior frontal gyrus (SFG) and right superior temporal gyrus (STG).

TABLE 3: Brain regions with significant group differences in normalized CBF with GMV correction.

\begin{tabular}{ccccc}
\hline $\begin{array}{l}\text { Brain } \\
\text { regions }\end{array}$ & BA & $\begin{array}{c}\text { Peak MNI coordinates } \\
x, y, z(\mathrm{~mm})\end{array}$ & $\begin{array}{c}\text { Peak } T \\
\text { value }\end{array}$ & $\begin{array}{c}\text { Cluster size } \\
\text { (voxels) }\end{array}$ \\
\hline mTBI > HC & & & & \\
\multicolumn{1}{c}{ L_ITG } & 20 & $-42,-14,-20$ & 4.717 & 262 \\
R_ITG & 20 & $46,-18,-20$ & 5.286 & 545 \\
mTBI < HC & & & & \\
L_SFG & 10 & $-18,56,20$ & -4.984 & 231 \\
R_SFG & 10 & $24,54,22$ & -5.588 & 455 \\
R_STG & 44 & $62,6,6$ & -4.348 & 163 \\
\hline
\end{tabular}

Thresholds were set at a corrected $p<0.01$ corrected by FDR criterion. MNI: Montreal Neurological Institute; mTBI: mild traumatic brain injury; HC: healthy control; ITG: inferior temporal gyrus; SFG: superior frontal gyrus; STG: superior temporal gyrus.

with decreased CBF in the right superior temporal gyrus (STG) were identified after GMV correction.

3.3. CBF Connectivity Patterns. A total of six ROIs were defined as the seed regions that had significant CBF differences between the mTBI patients and HC subjects. The $\mathrm{CBF}$ connectivity maps of each ROI from the two groups

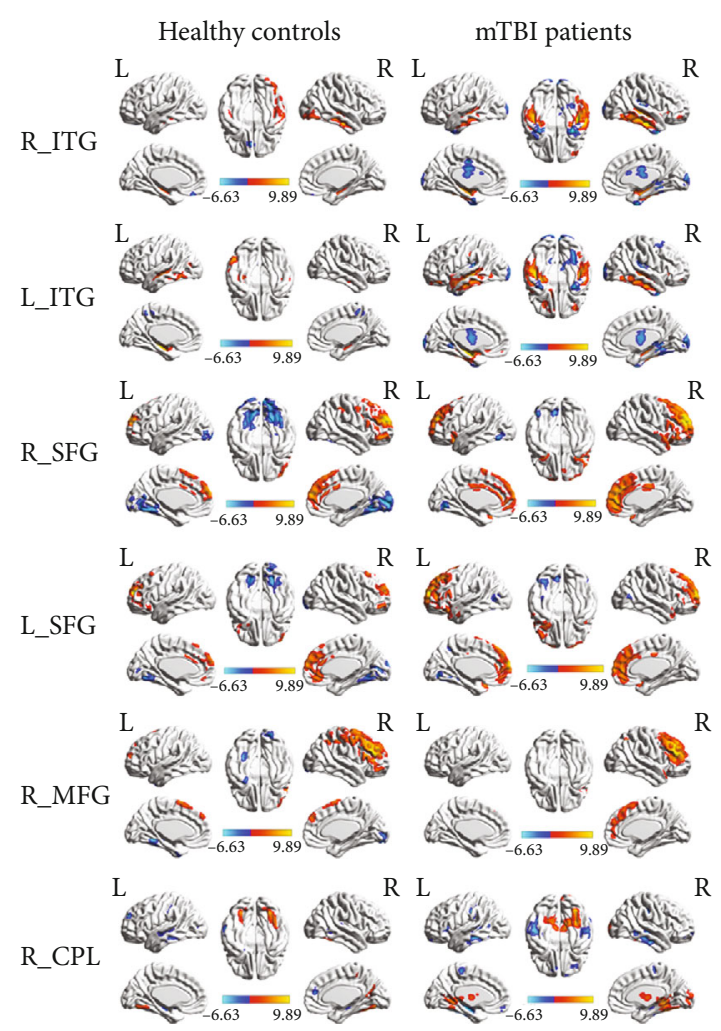

Figure 3: The CBF connectivity maps of six ROIs from the mTBI patients and the HC were displayed, including the bilateral inferior temporal gyrus (ITG), bilateral superior frontal gyrus (SFG), right middle frontal gyrus (MFG), and right cerebellum posterior lobe (CPL).

are displayed in Figure 3. The left SFG demonstrated similar positive connectivity in both groups but exhibited completely different negative connectivity patterns. The right SFG had $\mathrm{CBF}$ connectivity with near regions in the controls, but it exhibited more extensive positive connectivity in mTBI patients. The right CPL showed CBF connectivity with the regions that mainly located in the bilateral cerebral hemisphere in the HC but exhibited more extensive positive and negative connectivity patterns in the mTBI patients. The right MFG had CBF connectivity with near regions in the $\mathrm{HC}$, but it showed more extensive positive connectivity and different negative connectivity patterns. However, the right ITG and the left ITG had a similar connectivity pattern in both groups.

3.4. Group Differences in CBF Connectivity. Group differences in CBF connectivity are shown in Figure 4 and Table 4. Compared with HC, the acute mTBI patients exhibited decreased negative CBF connectivity between the seed ROI of the right CPL and the right ITG as well as the left ITG, but these patients also showed increased positive CBF connectivity between the seed of right CPL and the right FG. Compared with HC subjects, acute mTBI patients also showed decreased negative CBF connectivity between the seed ROI of the left SFG and the left MOG. In addition, acute mTBI patients exhibited increased negative CBF connectivity 


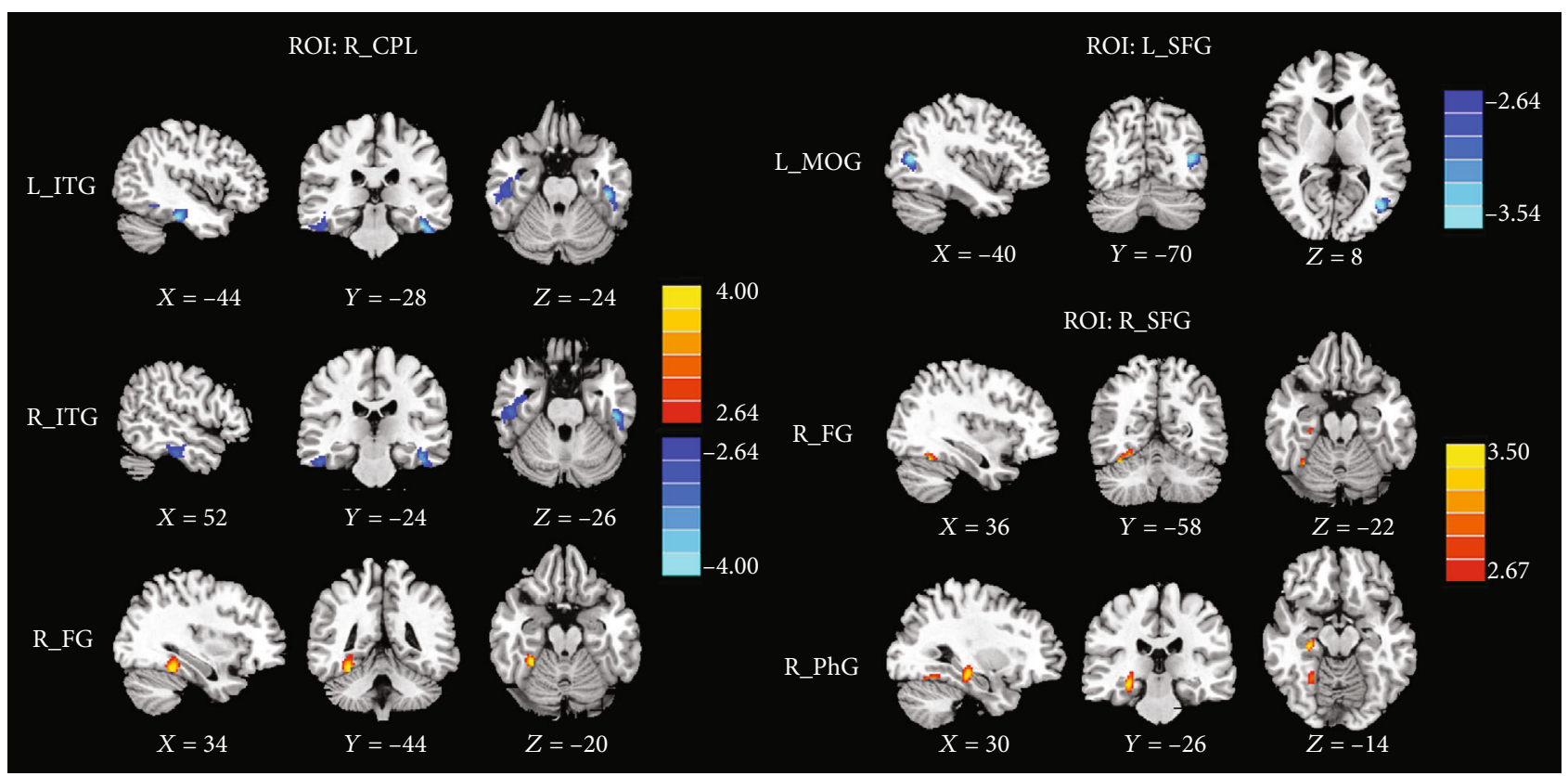

FIgure 4: Compared with HC, the acute mTBI patients exhibited decreased negative CBF connectivity between the seed ROI of the right CPL and the right ITG as well as the left ITG and increased positive CBF connectivity with the right FG; the acute mTBI patients showed decreased negative CBF connectivity between the seed ROI of the left SFG and the left MOG; the acute mTBI patients exhibited increased negative CBF connectivity between the seed ROI of the right SFG and the right FG as well as the right PhG.

TABLE 4: Brain regions with significant group differences in CBF connectivity.

\begin{tabular}{lcccc}
\hline ROI & Brain regions & BA & Peak MNI coordinates $x, y, z(\mathrm{~mm})$ & Peak T value \\
\hline \multirow{2}{*}{ R_CPL } & L_ITG & 20 & $-44,-28,-24$ & -3.941 \\
& R_ITG & 20 & $52,-24,-26$ & -3.296 \\
\multirow{2}{*}{ L_SFG } & R_FG & 37 & $34,-44,-20$ & 4.276 \\
& L_MOG & 39 & $-40,-70,8$ & -3.738 \\
R_SFG & R_FG & 37 & $36,-58,-22$ & 3.475 \\
& R_PhG & 36 & $30,-26,-14$ & 3.571 \\
\hline
\end{tabular}

Thresholds were set at a corrected $p<0.01$ corrected by FDR criterion. CBF: cerebral blood flow; ROI: region of interest; MNI: Montreal Neurological Institute; CPL: cerebellum posterior lobe; ITG: inferior temporal gyrus; FG: fusiform gyrus; SFG: superior frontal gyrus; MOG: middle occipital gyrus; PhG: parahippocampal gyrus.

between the seed ROI of the right SFG and the right FG as well as the right PhG. The seed ROI of the right MFG and the bilateral ITG in CBF did not exhibit any significant differences in the CBF connectivity between both groups.

\subsection{Correlations between CBF and Neurocognitive Outcome.} The significant correlations between the CBF changes and the neurocognitive outcome (assessed by MoCA) are depicted in Figure 5. Regarding the neurocognitive state, The MoCA scores and the visuospatial/execution scores were positively correlated with the normalized CBF of the left SFG, respectively $(r=0.487, p=0.001 ; r=0.355, p=0.018)$. In addition, the attention scores were negatively correlated with the normalized $\mathrm{CBF}$ of the right ITG in the acute mTBI patients $(r=-0.400, p=0.007)$. However, the other regional $\mathrm{CBF}$ values and CBF connectivity were not correlated with the cognitive scores.

\section{Discussion}

In the present study, we applied an ASL-MRI technique to investigate the normalized $\mathrm{CBF}$ and $\mathrm{CBF}$ connectivity changes in acute mTBI patients. To the best of our knowledge, this study is the first to use ASL-MRI to analyze the CBF connectivity patterns of acute mTBI patients. These patients had increased CBF in the bilateral ITG and decreased CBF in the bilateral SFG, right MFG, and CPL. The outcome of the cognitive functional assessment was correlated with the regional normalized CBF. The CBF of the left SFG and right ITG was correlated with the cognitive scores, respectively. More importantly, the CBF connectivity of the bilateral ITG and FG, right PhG, and left MOG was also impaired in acute mTBI patients.

We identified decreased CBF in the bilateral SFG and right MFG, the key regions of the prefrontal lobe, consisting 


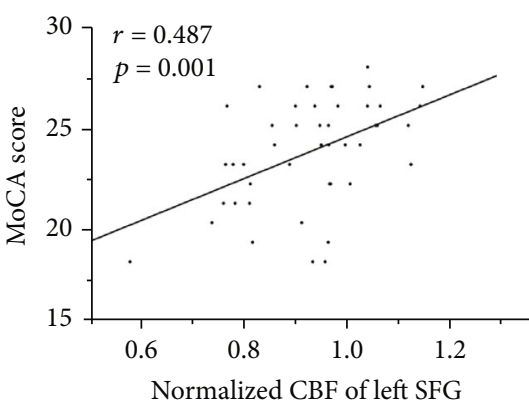

(a)

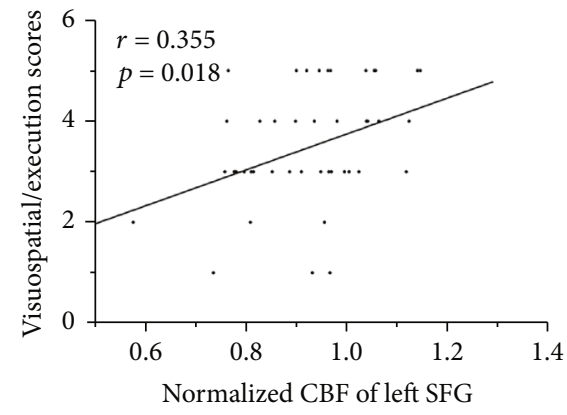

(b)

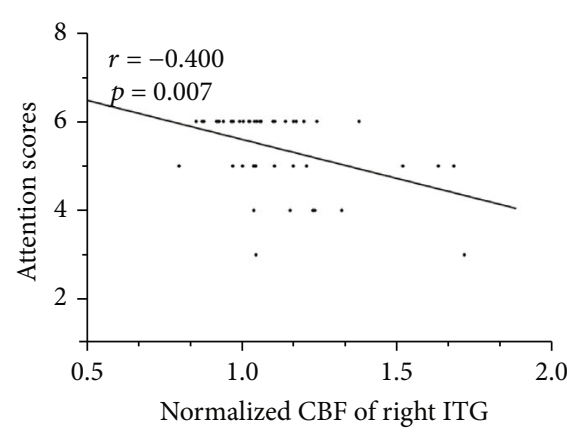

(c)

FIgURE 5: The significant correlations between the CBF changes and the neurocognitive outcome. The MoCA scores (a) and the visuospatial/execution scores (b) were positively correlated with the normalized CBF of the left SFG, respectively $(r=0.487, p=0.001$; $r=0.355, p=0.018$ ). The attention scores (c) were negatively correlated with the normalized CBF of the right ITG in the acute mTBI patients $(r=-0.400, p=0.007)$.

in prior studies in mTBI using ASL-MRI, PET, or SPECT [14, 30, 31]. The frontal lobes are highly evolved and regulate complex behaviors in healthy subjects, which is involved in complex cognitive functions such as planning complex cognitive behaviors, personality expression, decision-making, and regulating social behavior, so hypofrontality may contribute to the clinical symptoms in mTBI [32]. Meanwhile, our result showed that the CBF of the left SFG was positively correlated with the MoCA scores and the visuospatial/execution scores, suggesting that the hypoperfusion in the left $\mathrm{CBF}$ may contribute to cognitive deficits in the acute mTBI. In the present study, we also identified decreased $\mathrm{CBF}$ in the right $\mathrm{CPL}$, which is involved in the cerebellum network. Although the cerebellum is primarily involved in the function of the motor actions and control, recent studies suggested that the cerebellum is also involved in multiple functions, including cognitive and affective processing, motor-related processing, the experience of thirst, and pain-related processes [33]. Using SPECT, Micarelli et al. have demonstrated that mTBI patients at the acute stage had abnormal CBF in specific regions of the cerebellum, and altered cerebellar CBF was linked to cognitive function [34], confirming the involvement of cerebellum in mTBI. In contrast with the above results, we did not identify any significant correlation between the CBF values in the region of cerebellum and the cognitive scores. The possible reason for this heterogeneous result may result from the differences among the subjects and the experimental methods. Nevertheless, the association of the cerebellum with cognitive impairment after mTBI has not been substantially elucidated and needs to be corroborated in future studies.

In line with previous findings $[8,15]$, we also identified increased CBF in the bilateral ITG in acute mTBI patients. The ITG is involved in the processes of multimodal sensory integration and visual perception and has been demonstrated volumetric atrophy in mTBI [35]. One possible explanation for the increased CBF in the temporal lobe may result from a short-term compensatory mechanism during the first few days' postinjury, although it has been postulated that the trauma of brain alters cerebrovascular reactivity within the brain. Specially, the increased CBF in the right ITG was found to be negatively correlated with the attention scores in acute mTBI patients, which may explain the deficits of some cognitive function in mTBI. Contrarily, Newberg et al. identified significantly lower CBF levels in the right temporal lobes of mTBI patients using SPECT [36]. In addition, one study found that children with mTBI with persistent posttraumatic symptoms had more severe medial temporal lobe hypoperfusion than those without persistent posttraumatic symptoms [37]. All these studies suggested that the altered CBF in the ITG may underlie the deficits in some cognitive function or posttraumatic symptoms in mTBI. Nevertheless, the specific clinical implication for the altered CBF in the ITG is unclear and needs to be further investigated.

In order to eliminate the effect of GMV, we repeated the voxel-based CBF analyses corrected for the GMV of each voxel. After GMV correction, the mTBI patients at the acute stage exhibited significantly increased CBF in the bilateral ITG and decreased CBF in the bilateral SFG, which were the same as the regions without GMV correction. These findings indicate that the changes of $\mathrm{CBF}$ in these regions may be independent of changes in GMV. However, the original cluster with reduced CBF in the right CPL disappeared and a new cluster with decreased CBF in the STG were identified after GMV correction, suggesting that the changes of CBF in the two regions may be related to GM atrophy, either secondary to GM atrophy or compensated for by GM atrophy.

Connectivity alterations in mTBI patients have been extensively studied by MRI techniques, such as the anatomical connectivity derived from diffusion tensor MRI, the structural connectivity derived from structural MRI, and the functional connectivity derived from functional MRI. However, to our knowledge, no study has investigated the CBF connectivity in mTBI. Despite both blood-oxygen leveldependent (BOLD) connectivity and CBF connectivity measure functional correlations between brain regions, they are calculated by different methods and also represent different physiological meanings. The BOLD connectivity was obtained by measuring the temporal correlation of BOLD signal fluctuations between individual brain regions. However, the CBF connectivity was obtained by calculating the correlation coefficient of CBF between brain regions of a group of individuals [38]. For a group of individuals, we 
can get multiple BOLD connectivity values (a value for an individual), but only one $\mathrm{CBF}$ connectivity value could be obtained. The BOLD connectivity represents the synchronization of neural activity between brain regions, while $\mathrm{CBF}$ connectivity reflects the coordination of perfusion or metabolism between brain regions. It is worth pointing out that, compared with BOLD connectivity affected by cerebral blood volume, cerebral oxygen metabolism rate, and other physiological parameters, CBF connectivity is only regulated by regional $\mathrm{CBF}$, which has a clearer physiological implication [19]. In the current study, we identified CBF disconnections between the left SFG and the left MOG and between the seed ROI of the right SFG and the right FG as well as the right $\mathrm{PhG}$. The MOG and FG are visual network brain regions, and the $\mathrm{PhG}$ is an important structure of hippocampal function, whose damage can cause abnormal emotional and cognitive behaviors [39]. Thus, the disconnection between bilateral SFG and these regions may be linked with the functional deficits in cognitive integration in the acute mTBI patients. Some recent resting-state fMRI BOLD connectivity studies has identified disconnection functional coupling between the prefrontal cortex and these regions [40, 41], which are consistent with our results. In addition, the CBF disconnection between the right CPL and bilateral ITG as well as the right FG was also been identified in mTBI patients, suggesting a disruption of the cerebellar-subcortical-cortical loop, which is partly consistent with the previous restingstate functional connectivity studies that indicate the CPL-FG disconnection and the abnormality of the cerebellum network in mTBI [42-44]. The disconnection of the cerebellum may be related to the functional deficits in cognitive integration in acute mTBI patients. These findings suggested that the $\mathrm{CBF}$ connectivity alterations in $\mathrm{CPL}$ may be one important brain characteristic of mTBI at the acute stage.

Several limitations should be acknowledged in the current study. First, due to the strict inclusion and exclusion criteria, we recruited a relatively small sample, which may affect the statistical reliability of our results. We hope to expand the sample size and obtain more reliable results in the future studies. Second, all the acute mTBI patients enrolled in this study suffered from complicated injury mechanisms that will influence our interpretation. Subgroup analysis based on different injury mechanisms may help to more accurately describe the pathogenesis of cognitive impairment in patients with mTBI. Furthermore, the CBF connectivity was calculated by analyzing interregional CBF correlations between subject groups (with only one $\mathrm{CBF}$ connectivity value for each group) and cannot be used to compare connectivity differences between any two subjects because CBF connectivity is a group-level measure, not an individual-level measure. This measure cannot be used to analyze correlations with clinical or cognitive parameters. The development of ASL techniques with higher temporal resolution and the calculation of CBF connectivity may help solve this problem. Finally, only the brain regions with significant differences in $\mathrm{CBF}$ between groups were selected as ROIs for CBF connectivity analysis, which may lead to the omission of $\mathrm{CBF}$ connectivity changes between brain regions with normal $\mathrm{CBF}$ values. A data-driven approach to wholebrain CBF connectivity analysis will be considered in our future study.

In summary, this study identified the CBF changes in multiple cortical and subcortical regions using ASL-MRI, which may underlie deficits in cognitive function of mTBI patients at the acute stage. Disconnection of CBF connectivity of the bilateral SFG and the right CPL in acute mTBI was investigated for the first time. In our study, both the changes in regional CBF and the disorder of CBF connectivity highlight the need to study the underlying neuropathology of cognitive impairment of the acute mTBI patients from the perspective of the regional and interregional characteristics of the resting-stage CBF.

\section{Data Availability}

The data used to support the findings of this study are available from the corresponding author upon request.

\section{Conflicts of Interest}

The authors declare that there is no potential conflict of interests regarding the publication of this paper.

\section{Authors' Contributions}

FL and LL contributed to the data collection and data analyses and wrote the paper. SS, HC, PW, and NAH helped revise the paper. XY and Y-CC designed the experiment and revised the paper. Fengfang Li and Liyan Lu contributed equally to this work.

\section{Acknowledgments}

This work was supported by the Jiangsu Provincial Special Program of Medical Science (No. BE2017614), Youth Medical Talents of Jiangsu Province (No. QNRC2016062), and the 333 High-Level Talents Training Project of Jiangsu Province (No. BRA2019122).

\section{References}

[1] Y. Hannawi and R. D. Stevens, "Mapping the connectome following traumatic brain injury," Current Neurology and Neuroscience Reports, vol. 16, no. 5, 2016.

[2] S. Polinder, M. C. Cnossen, R. G. L. Real et al., "A Multidimensional Approach to Post-concussion Symptoms in Mild Traumatic Brain Injury," Frontiers in Neurology, vol. 9, 2018.

[3] M. G. de Freitas Cardoso, R. M. Faleiro, J. J. de Paula et al., "Cognitive impairment following acute mild traumatic brain injury," Frontiers in Neurology, vol. 10, 2019.

[4] P. Santhanam, S. H. Wilson, T. R. Oakes, and L. K. Weaver, "Effects of mild traumatic brain injury and post-traumatic stress disorder on resting-state default mode network connectivity," Brain Research, vol. 1711, pp. 77-82, 2019.

[5] K. Kenney, F. Amyot, M. Haber et al., "Cerebral vascular injury in traumatic brain injury," Experimental Neurology, vol. 275, Part 3, pp. 353-366, 2016. 
[6] J. A. Stephens, P. Liu, H. Lu, and S. J. Suskauer, "Cerebral blood flow after mild traumatic brain injury: associations between symptoms and post-injury perfusion," Journal of Neurotrauma, vol. 35, no. 2, pp. 241-248, 2018.

[7] K. M. Barlow, L. D. Marcil, D. Dewey et al., "Cerebral perfusion changes in post-concussion syndrome: a prospective controlled cohort study," Journal of Neurotrauma, vol. 34, no. 5, pp. 996-1004, 2017.

[8] B. Pöttker, F. Stöber, R. Hummel et al., "Traumatic brain injury causes long-term behavioral changes related to regionspecific increases of cerebral blood flow," Brain Structure and Function, vol. 222, no. 9, pp. 4005-4021, 2017.

[9] T. Crider, D. Eng, P. R. Sarkar, J. Cordero, J. C. Krusz, and S. N. Sarkar, "Microvascular and large vein abnormalities in young patients after mild head trauma and associated fatigue: A brain SPECT evaluation and posture dependence modeling," Clinical Neurology and Neurosurgery, vol. 170, pp. 159-164, 2018.

[10] J. B. Andre, “Arterial Spin Labeling Magnetic Resonance Perfusion for Traumatic Brain Injury Technical Challenges and Potentials," Topics in Magnetic Resonance Imaging, vol. 24, no. 5, pp. 275-287, 2015.

[11] M. L. Ho, “Arterial spin labeling: Clinical applications," Journal of Neuroradiology, vol. 45, no. 5, pp. 276-289, 2018.

[12] V. C. Keil, N. S. Hartkamp, D. J. A. Connolly et al., "Added value of arterial spin labeling magnetic resonance imaging in pediatric neuroradiology: pitfalls and applications," Pediatric Radiology, vol. 49, no. 2, pp. 245-253, 2019.

[13] M. Haber, F. Amyot, K. Kenney et al., "Vascular abnormalities within normal appearing tissue in chronic traumatic brain injury," Journal of Neurotrauma, vol. 35, no. 19, pp. 22502258, 2018.

[14] Y. Wang, J. D. West, J. N. Bailey et al., "Decreased Cerebral Blood Flow in Chronic Pediatric Mild TBI: An MRI Perfusion Study," Developmental Neuropsychology, vol. 40, no. 1, pp. 4044,2015

[15] J. Hamer, N. W. Churchill, M. G. Hutchison, S. J. Graham, and T. A. Schweizer, "Sex differences in cerebral blood flow associated with a history of concussion," Journal of Neurotrauma, vol. 37, no. 10, pp. 1197-1203, 2020.

[16] Y. Ge, M. B. Patel, Q. Chen et al., “Assessment of thalamic perfusion in patients with mild traumatic brain injury by true FISP arterial spin labelling MR imaging at 3T," Brain Injury, vol. 23, no. 7-8, pp. 666-674, 2009.

[17] A. E. Leeuwis, M. R. Benedictus, J. P. A. Kuijer et al., "Lower cerebral blood flow is associated with impairment in multiple cognitive domains in Alzheimer's disease," Alzheimer's \& Dementia, vol. 13, no. 5, pp. 531-540, 2017.

[18] I. Havsteen, J. Damm Nybing, H. Christensen, and A. F. Christensen, "Arterial spin labeling: a technical overview," Acta Radiologica, vol. 59, no. 10, pp. 1232-1238, 2018.

[19] L. Melie-García, G. Sanabria-Diaz, and C. Sánchez-Catasús, "Studying the topological organization of the cerebral blood flow fluctuations in resting state," NeuroImage, vol. 64, pp. 173-184, 2013.

[20] P. Jezzard, M. A. Chappell, and T. W. Okell, "Arterial spin labeling for the measurement of cerebral perfusion and angiography," Journal of Cerebral Blood Flow \& Metabolism, vol. 38, no. 4, pp. 603-626, 2017.

[21] L. B. Cui, L. X. Wang, P. Tian et al., "Aberrant perfusion and its connectivity within default mode network of first-episode drug-naïve schizophrenia patients and their unaffected first- degree relatives," Scientific Reports, vol. 7, no. 1, article 16201, 2017.

[22] K. Jann, R. X. Smith, E. A. Rios Piedra, M. Dapretto, and D. J. J. Wang, "Noise reduction in arterial spin labeling based functional connectivity using nuisance variables," Frontiers in Neuroscience, vol. 10, 2016.

[23] Z. S. Nasreddine, N. A. Phillips, V Ã.@r. BÃ@dirian et al., "The Montreal Cognitive Assessment, MoCA: A Brief Screening Tool For Mild Cognitive Impairment," Journal of the American Geriatrics Society, vol. 53, no. 4, pp. 695-699, 2005.

[24] E. de Guise, A. Y. Alturki, J. LeBlanc et al., "The Montreal Cognitive Assessment in Persons with Traumatic Brain Injury," Applied Neuropsychology: Adult, vol. 21, no. 2, pp. 128-135, 2013.

[25] Z. Wang, "Improving cerebral blood flow quantification for arterial spin labeled perfusion MRI by removing residual motion artifacts and global signal fluctuations," Magnetic Resonance Imaging, vol. 30, no. 10, pp. 1409-1415, 2012.

[26] J. Zhu, C. Zhuo, W. Qin et al., "Altered resting-state cerebral blood flow and its connectivity in schizophrenia," Journal of Psychiatric Research, vol. 63, pp. 28-35, 2015.

[27] G. Xu, H. A. Rowley, G. Wu et al., "Reliability and precision of pseudo-continuous arterial spin labeling perfusion MRI on 3.0 T and comparison with ${ }^{15} \mathrm{O}$-water PET in elderly subjects at risk for Alzheimer's disease," NMR in Biomedicine, vol. 23, no. 3, 2010.

[28] S. Aslan and H. Lu, "On the sensitivity of ASL MRI in detecting regional differences in cerebral blood flow," Magnetic Resonance Imaging, vol. 28, no. 7, pp. 928-935, 2010.

[29] J. Ashburner, "A fast diffeomorphic image registration algorithm," NeuroImage, vol. 38, no. 1, pp. 95-113, 2007.

[30] N. W. Churchill, M. G. Hutchison, S. J. Graham, and T. A. Schweizer, "Symptom correlates of cerebral blood flow following acute concussion," NeuroImage Clinical, vol. 16, pp. 234239, 2017.

[31] C. Möller, L. E. Nordin, A. Bartfai, P. Julin, and T.-Q. Li, "Fatigue and Cognitive Fatigability in Mild Traumatic Brain Injury are Correlated with Altered Neural Activity during Vigilance Test Performance," Frontiers in Neurology, vol. 8, 2017.

[32] A. B. Dodd, H. Lu, C. J. Wertz et al., "Persistent alterations in cerebrovascular reactivity in response to hypercapnia following pediatric mild traumatic brain injury," Journal of Cerebral Blood Flow \& Metabolism, p. 0271678X1989688, 2020.

[33] J. Diedrichsen, M. King, C. Hernandez-Castillo, M. Sereno, and R. B. Ivry, "Universal Transform or Multiple Functionality? Understanding the Contribution of the Human Cerebellum across Task Domains," Neuron, vol. 102, no. 5, pp. 918-928, 2019.

[34] A. Micarelli, H. Jacobsson, S. A. Larsson, C. Jonsson, and M. Pagani, "Neurobiological insight into hyperbaric hyperoxia," Acta Physiologica, vol. 209, no. 1, pp. 69-76, 2013.

[35] R. De Pauw, I. Coppieters, K. Caeyenberghs et al., "Associations between brain morphology and motor performance in chronic neck pain: A whole-brain surface-based morphometry approach," Human Brain Mapping, vol. 40, no. 14, pp. 42664278, 2019.

[36] A. B. Newberg, M. Serruya, A. Gepty et al., "Clinical Comparison of ${ }^{99 \mathrm{~m}} \mathrm{Tc}$ Exametazime and ${ }^{123} \mathrm{I}$ Ioflupane SPECT in Patients with Chronic Mild Traumatic Brain Injury," PLoS One, vol. 9, no. 1, article e87009, 2014. 
[37] D. Agrawal, N. K. Gowda, C. S. Bal, M. Pant, and A. K. Mahapatra, "Is medial temporal injury responsible for pediatric postconcussion syndrome? A prospective controlled study with single-photon emission computerized tomography," Journal of Neurosurgery, vol. 102, no. 2, pp. 167-171, 2005.

[38] B. Biswal, F. Z. Yetkin, V. M. Haughton, and J. S. Hyde, "Functional connectivity in the motor cortex of resting human brain using echo-planar mri," Magnetic Resonance in Medicine, vol. 34, no. 4, pp. 537-541, 1995.

[39] N. A. Herweg, A. D. Sharan, M. R. Sperling, A. Brandt, A. Schulze-Bonhage, and M. J. Kahana, "Reactivated Spatial Context Guides Episodic Recall," The Journal of Neuroscience, vol. 40, no. 10, pp. 2119-2128, 2020.

[40] K. Lancaster, U. M. Venkatesan, J. Lengenfelder, and H. M. Genova, "Default Mode Network Connectivity Predicts Emotion Recognition and Social Integration After Traumatic Brain Injury," Frontiers in Neurology, vol. 10, 2019.

[41] S. B. Rockswold, P. C. Burton, A. Chang et al., "Functional magnetic resonance imaging and oculomotor dysfunction in mild traumatic brain injury," Journal of Neurotrauma, vol. 36, no. 7, pp. 1099-1105, 2019.

[42] M. R. Newsome, A. R. Mayer, X. Lin et al., "Chronic effects of blast-related TBI on subcortical functional connectivity in veterans," Journal of the International Neuropsychological Society, vol. 22, no. 6, pp. 631-642, 2016.

[43] S. Wang, L. Hu, J. Cao et al., "Sex Differences in Abnormal Intrinsic Functional Connectivity After Acute Mild Traumatic Brain Injury," Frontiers in Neural Circuits, vol. 12, 2018.

[44] F. Li, L. Lu, H. Chen et al., "Disrupted brain functional hub and causal connectivity in acute mild traumatic brain injury," Aging, vol. 11, no. 22, pp. 10684-10696, 2019. 